Issued by Sandia National Laboratories, operated for the United States Department of Energy by Sandia Corporation.

NOTICE: This report was prepared as an account of work sponsored by an agency of the United States Government. Neither the United States Government nor any agency thereof, nor any of their employees, nor any of their contractors, subcontractors, or their employees, makes any warranty, express or implied, or assumes any legal liability or responsibility for the accuracy, completeness, or usefulness of any information, apparatus, product, or process disclosed, or represents that its use would not infringe privately owned rights. Reference herein to any specific commercial product, process, or service by trade name, trademark, manufacturer, or otherwise, does not necessarily constitute or imply its endorsement, recommendation, or favoring by the United States Government, any agency thereof or any of their contractors or subcontractors. The views and opinions expressed herein do not necessarily state or reflect those of the United States Government, any agency thereof or any of their contractors.

Printed in the United States of America. This report has been reproduced directly from the best available copy.

Available to DOE and DOE contractors from

Office of Scientific and Technical Information

PO Box 62

Oak Ridge, TN 37831

Prices available from (615) 576-8401, FTS 626-8401

Available to the public from

National Technical Information Service

US Department of Commerce

5285 Port Royal RD

Springfield, VA 22161

NTIS price codes

Printed copy: A03

Microfiche copy: A06 


\section{DISCLAIMER}

Portions of this document may be illegible in electronic image products. Images are produced from the best available original document. 


\title{
Assembly Partitioning with a Constant Number of Translations
}

\author{
Dan Halperin* \\ Robotics Laboratory \\ Department of Computer Science \\ Stanford University \\ Stanford, CA 94305 \\ Randall H. Wilson \\ Intelligent Systems and Robotics Center \\ Sandia National Laboratories \\ Albuquerque, NM 87185
}

August 24, 1994

\begin{abstract}
We consider the following problem that arises in assembly planning: given an assembly, identify a subassembly that can be removed as a rigid object without disturbing the rest of the assembly. This is the assembly partitioning problem. Specifically, we consider planar assemblies of simple polygons and subassembly removal paths consisting of a single finite translation followed by a translation to infinity. We show that such a subassembly and removal path can be determined in $O\left(n^{1.46} N^{6}\right)$ time, where $n$ is the number of polygons in the assembly and $N$ is the total number of edges and vertices of all the parts together. We then extend this formulation to removal paths consisting of a small number of finite translations, followed by a translation to infinity. In this case the algorithm runs in time polynomial in the number of parts, but exponential in the number of translations a path may contain.
\end{abstract}

\section{MASTEF}

${ }^{*}$ Work on this paper by the first author has been supported by a grant from the Stanford Integrated Manufacturing Association (SIMA), by NSF/ARPA Grant IRI-9306544, and by NSF Grant CCR-9215219. This research was performed while the second author was at Stanford University, and has been supported by the Stanford Integrated Manufacturing Association (SIMA). 


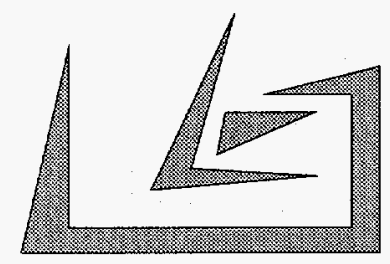

(a)

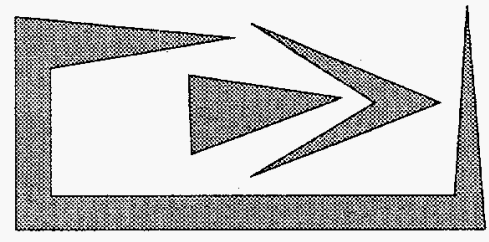

(b)

Figure 1: Assemblies requiring non-straight-line motions for disassembly. (a) is a monotone binary assembly while (b) is not.

\section{Introduction}

This paper addresses a geometric problem in assembly planning. The problem is: given an assembly of parts, identify a subassembly that can be removed from the assembly. That is, identify a proper subset of the parts that can be moved (as a single rigid object) to infinity without disturbing the other parts. This is the assembly partitioning problem.

The partitioning problem arises in assembly planning. An assembly sequence is a sequence of motions that constructs an assembly from its constituent parts, and for rigid parts is the reverse of a disassembly sequence. In this paper we assume that the (dis)assembly sequences are binary and monotone, i.e., only one group of parts moves at a time, and the motion completely separates the moved parts from the rest of the assembly. In other words, no parts are placed in intermediate positions. For example, the assembly in Figure 1(a) can be assembled by a monotone binary assembly sequence involving only translations (the two small parts are placed together then inserted into the larger), while the assembly in Figure 1(b) cannot. A monotone binary assembly sequence can be found, if one exists for the assembly, by repeated application of an assembly partitioning algorithm.

A large majority of assemblies in industry are monotone binary, and most assembly planning systems make this assumption as well [8]. As a result, the partitioning problem is of great practical importance.

Previous work has presented polynomial-time partitioning algorithms when the motion used to remove the subassembly must be a single translation to infinity $[1,18]$. In addition, it has been shown that when the removal motion may consist of any number of translations, partitioning is NP-complete [10].

In this paper we consider the case where a sequence of two translations may be used to remove a subassembly. We restrict our attention to planar assemblies of simple polygons. We give a polynomial-time algorithm to determine whether an assembly of non-overlapping simple polygons can be partitioned by a motion consisting of a finite translation followed by a translation to infinity. A subassembly and its removal motion can be identified in the same time bound. In addition, we extend this result to removal motions consisting of $k$ translations; in this case the algorithm runs in time exponential in $k$.

The rest of the paper is organized as follows. In Section 2 we survey some related work. In Section 3 we present the basic necessary terminology together with an initial analysis of our solution approach. In Section 4 we present our solution to the two-translation problem, which we then extend to the case of a bounded number of translations in Section 5 . Some 
concluding remarks and open problems are given in Section 6.

\section{Related Work}

For an overview of geometric separation problems see Toussaint [17]. Pollack, Sharir, and Sifrony [16] give an efficient algorithm to separate two simple polygons in the plane.

Polynomial-time solutions to the binary partitioning problem exist for certain types of restricted disassembly motions. Arkin, Connelly, and Mitchell [1] give a polynomial-time algorithm for partitioning an assembly of polygons in the plane with a single infinite translation, and they have extended the algorithm to polyhedral assemblies in $3 \mathrm{D}$ [14]. Wilson [18, 19] solves the $3 \mathrm{D}$ partitioning problem for polyhedra in polynomial time when the disassembly motions are restricted to either (a) infinitesimal translations and rotations or (b) infinite translations. In the infinitesimal case, a subassembly is identified that can move a very small distance, which is only a necessary condition on a removal path.

Lozano-Pérez and Wilson [13] extend Wilson's framework to arbitrary removal motions. While their algorithm might prove useful in practice, Kavraki, Latombe, and Wilson [10] show that for assemblies of polygons in the plane the problem of partitioning for arbitrary removal motions is NP-complete.

This paper addresses an intermediate case for assemblies of simple polygons in the plane. In this intermediate case the number of translations allowed to remove a subassembly is greater than one, but bounded by some constant $k$. We first consider the case where $k=2$, and then consider the case $k>2$.

\section{Preliminaries: Terminology and Initial Analysis}

In this section we present some of the terminology that we will be using throughout the paper, together with an initial analysis of the problem we are considering.

\subsection{Arrangements of Curves and of Surfaces}

An arrangement of curves in the plane is the subdivision of the plane induced by these curves. Consider, for example, the arrangement induced by a collection of $n$ lines in the plane. An arrangement of lines partitions the plane into vertices, edges and faces. A vertex is an intersection point of two lines, an edge is a maximal connected portion of a line that does not meet any vertex, and a face is a maximal connected region of the plane not meeting any edge or vertex. If we assume that the lines are in general position, namely, no two are parallel and no more than two meet at a single point, then it can be shown that the number of vertices (as well as the number of edges or faces) in the arrangement is $\Theta\left(n^{2}\right)$. Similarly, in three dimensions, we may consider an arrangement of $n$ planes, which partitions space into vertices, edges, faces and cells.

Arrangements are defined for non-linear objects as well, and the concept extends to higher dimensional Euclidean spaces. We will discuss arrangements of (hyper)surfaces or surface patches in $d$-dimensional Euclidean space, where the surfaces are assumed to be algebraic of bounded maximum degree, and bounded by algebraic surfaces of maximum constant degree. See [5] for detailed discussions on arrangements of lines and of hyperplanes in higher dimensional spaces. For discussion of arrangements of curves and surfaces (not necessarily linear) 
see, e.g., [6], [7]; in particular, the thesis [7] focuses on arrangements of surfaces in 3-space that are induced by motion planning problems and are closely related to the arrangements discussed in Section 4 of this paper.

We will often refer to two quantities regarding arrangements of $n$ surfaces in $d$-space: The complexity of the entire arrangement, and the time complexity of computing the entire arrangement. The complexity of an arrangement of $n$ surface patches in $d$-space is defined as the overall number of cells of dimension $k, 0 \leq k \leq d$, in the arrangement. It is well known that the maximum complexity $K_{d}(n)$ of an arrangement of $n$ surface patches in $d$-space as defined above is $\Theta\left(n^{d}\right)$ (see, e.g., [15]).

As for computing an arrangement of $n$ surfaces in $d$-space, we should first be more explicit as to' what we mean by "computing" an arrangement. For our purposes, we need a way to visit all cells in the arrangement, at each step moving from a cell to one of its neighbors. We will denote the worst-case running time of the best known algorithm for solving this problem by $T_{d}(n)$, where $d$ is the dimension of the space, and the maximum is taken over all arrangements of $n$ surfaces as defined above. For arrangements of lines in the plane, the running time of such an algorithm is $\Theta\left(n^{2}\right)$ (see, e.g., [5]). In fact, $T_{2}(n)$ is roughly quadratic, i.e., there is an algorithm with near-quadratic running time to compute the arrangement induced by any collection of $n$ algebraic curves of bounded degree. In higher dimensions, there are also algorithms to solve this problem with running time that is close to the worst case combinatorial complexity of the entire arrangement. Basu et al. [2] give an algorithm to find a point in every cell in a $d$-dimensional arrangement, in time $O\left(n^{d}\right)$, where $d$ is fixed, and where we assume that the algebraic degree of the surfaces is bounded (their result explicitly handles the dependence on this degree). It may be more convenient for our purpose, though, to have an algorithm that produces these sample points in a systematic way, namely, in a way that for most pairs of successive points that the algorithm produces they lie in adjacent cells. This can be done with roughly the same running time, using road-map like methods [3]. In any case, we will use the notation $T_{d}(n)$ as defined above rather than be specific about this bound.

\subsection{The Minkowski Sum of Two Polygons, Envelopes and Shadows}

Let $P$ and $Q$ be two sets in $R^{2}$. The Minkowski sum (or vector sum) of $P$ and $Q$, denoted $P \oplus Q$, is the set $\{p+q \mid p \in P, q \in Q\}$. We will also use the notation $P \ominus Q=P \oplus(-Q)=$ $\{p-q \mid p \in P, q \in Q\}$. Choose a reference point $r$ rigidly attached to $Q$, and suppose that $Q$ is placed such that the reference point coincides with the origin. Then $P \ominus Q$ is the loci of placements of the reference point where $P \cap Q \neq \emptyset$. The Minkowski sum is a useful concept in robot motion planning and related areas [12], often called the configuration space obstacle, or $C$-obstacle: the set $Q$ will collide with $P$ under a rigid translational motion along a path $t$ exactly when the reference point $r$, moved along $t$, intersects $P \ominus Q$.

For the assembly partitioning problem that we are considering, we will confine ourselves to the Minkowski sum of polygonal sets, which is itself a polygonal set. Let $P$ and $Q$ be two simple polygons, and let $u$ and $v$ denote the number of vertices in $P$ and $Q$ respectively. It is well known that the combinatorial complexity of the boundary of $P \ominus Q$, namely the overall number of edges and vertices on the boundary of $P \ominus Q$, is $O\left(u^{2} v^{2}\right)$, and this bound is tight in the worst case (see, e.g., [9]). However, for our purposes, we only need to know the outer boundary of $P \ominus Q$. Since it is defined by at most $O(u v)$ segments, its complexity is at most $O(u v \alpha(u v))$ [16], where $\alpha(n)$ is the extremely slowly growing functional inverse 
of Ackermann's function. Figure 2(a) shows two polygons $P$ and $Q$, and Figure 2(b) their Minkowski sum $P \ominus Q$.

In our analysis, we will consider portions of the Minkowski sum of two polygons, which we will refer to as envelopes. Let $M$ denote the outer boundary of $P \ominus Q$. The boundary $M$ is a simple polygon. We define the central envelope of $M, E_{C}(M)$, to be the collection of points of $M$ first hit by rays from the origin. More precisely, if $\rho$ is a ray from the origin, we let $F(\rho, M)$ be the intersection point of $\rho$ and $M$ which is nearest to the origin; $F(\rho, M)$ is undefined if $\rho \cap M=\emptyset$. Then, $E_{C}(M)$ is the union of $F(\rho, M)$ over all rays through the origin where $F(\rho, M)$ is defined. Figure 2(c) shows the central envelope for the polygon in Figure 2(b).

Next we define the $\phi$-upper envelope of $M$. Let $\lambda$ be a directed line, whose angle with the positive $x$-direction is $\phi$. Let $G(\lambda, M)$ be the farthest point of intersection of $\lambda$ and $M$ in the direction of $\lambda$, if they indeed intersect. The $\phi$-upper envelope of $M, E_{\phi}(M)$ is defined to be the union of $G(\lambda, M)$ over all lines $\lambda$ whose angle with the positive $x$-direction is $\phi$, whenever $G(\lambda, M)$ is defined. If $\phi$ is $90^{\circ}$, then our definition of $E_{\phi}(M)$ is similar to the standard definition of upper envelopes (see, e.g., [6]). Figure 2(d) shows the $\phi$-upper envelope of the polygon in Figure 2(b) for $\phi=90^{\circ}$.

Corresponding to each of the two envelopes, we now define a shadow. The central shadow of $M, S_{C}(M)$, is the collection of points in the plane such that the line connecting them to the origin intersects the central envelope $E_{C}(M)$. Similarly, the $\phi$-shadow of $M, S_{\phi}(M)$, is the union of rays whose angle with the positive $x$-axis is $\phi+180^{\circ}$ and whose termini lie on the $\phi$-upper envelope $E_{\phi}(M)$. Figures $2(\mathrm{e})$ and 2(f) show the central shadow and $90^{\circ}$-shadow, respectively, of the polygon in Figure $2(\mathrm{~b})$.

We will use the central shadow and $\phi$-shadow of $P \ominus Q$ to reason about relative translations of $Q$ with respect to $P$. For an initial translation $(x, y)$ of $Q$, the point $(x, y)$ is inside the central shadow $S_{C}(P \ominus Q)$ exactly when $Q$ collides with $P$ along the translation $(x, y)$. When $Q$ is already at position $(x, y)$ and translated infinitely in direction $\phi$, it collides with $P$ if and only if $(x, y)$ is inside the $\phi$-shadow $S_{\phi}(P \ominus Q)$.

Let $P_{i}$ and $P_{j}$ be two parts in an assembly $A$, with $n_{i}$ and $n_{j}$ vertices respectively. As mentioned above the complexity of the outer boundary of $P_{i} \ominus P_{j}$ is $O\left(n_{i} n_{j} \alpha\left(n_{i} n_{j}\right)\right)$. The same bound also holds for each of the envelopes we have defined, and for the boundary of the corresponding shadows of $P_{i} \ominus P_{j}$. Now, the outer boundary of $P_{i} \ominus P_{j}$, as well as the two envelopes lie each in the union of a collection $S$ of $O\left(n_{i} n_{j}\right)$ line segments-the collection of segments underlying the Minkowski sum. Each of these segments is either the Minkowski sum of an edge of $P_{i}$ and a vertex of $-P_{j}$ or the Minkowski sum of a vertex of $P_{i}$ and an edge of $-P_{j}$. The boundary of each of the shadows also lies in a collection of $O\left(n_{i} n_{j}\right)$ segments, which is the collection $S$ augmented with additional rays extended from endpoints of some of the segments in $S$.

For the subsequent analysis, which aims at worst-case asymptotic time bound for the algorithm that we present, it is sufficient to deal with the appropriate raw collection of segments rather than with the boundary of $P_{i} \ominus P_{j}$. Hence, we state the following Lemma.

Lemma 3.1 Let $P_{i}$ and $P_{j}$ be two polygonal parts with $n_{i}$ and $n_{j}$ vertices respectively. The outer boundary $M$ of $P_{i} \ominus P_{j}$, the central envelope of $M$, the $\phi$-envelope of $M$ for any fixed $\phi$, the boundary of the central shadow and the boundary of the $\phi$-shadow for any fixed $\phi$, each lies in the union of $O\left(n_{i} n_{j}\right)$ segments. 


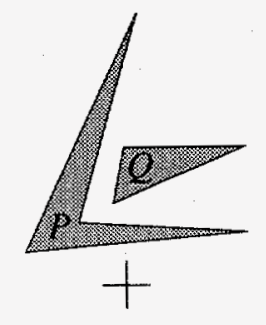

(a)

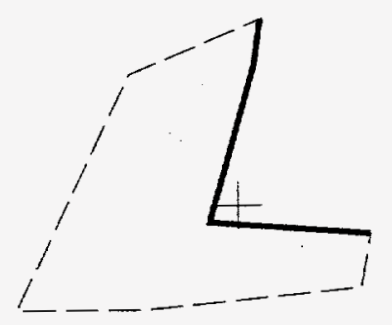

(c)

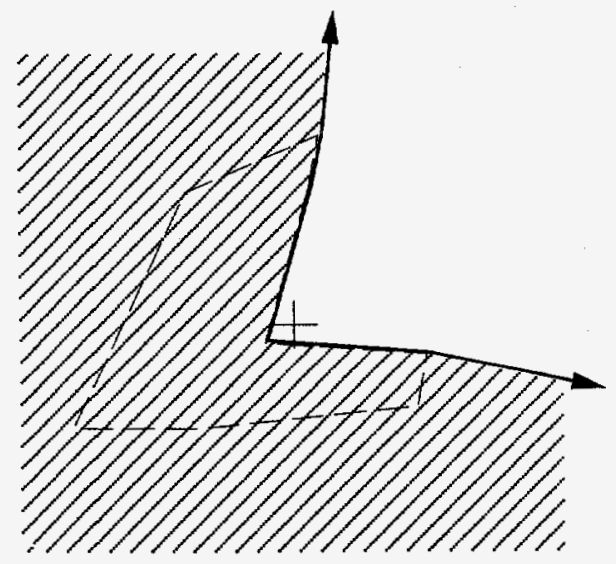

(e)

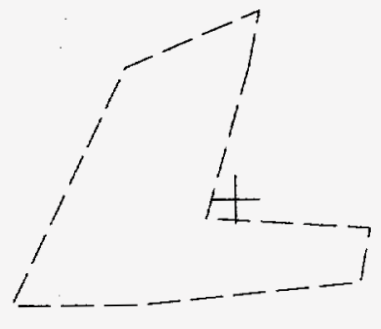

(b)

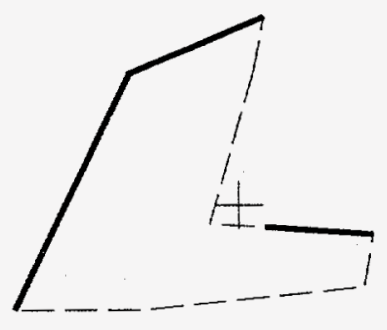

(d)

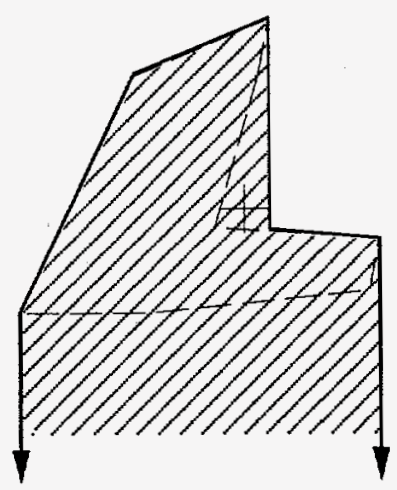

(f)

Figure 2: Some geometric preliminaries: (a) Two polygons $P$ and $Q$, (b) the boundary of the Minkowski sum of $P$ and $-Q, M=b d(P \ominus Q)$, (c) the central envelope $E_{C}(M)$, (d) the $\phi$-upper envelope $E_{90^{\circ}}(M)$, (e) the central shadow $S_{C}(M)$, and (f) the $\phi$-shadow $S_{90^{\circ}}(M)$. 


\subsection{Partitioning along a Single Path}

Consider an assembly $A$ of non-overlapping simple polygons in the plane. We will call the polygons parts, and will use the term subassembly loosely, to refer to a set of parts as well as their union. In general, we wish to identify a proper subassembly $S \subset A$ that can be completely separated from $A \backslash S^{1}$ by a collision-free rigid motion along a continuous path $t$. In this paper, we will confine ourselves to paths that are the concatenation of a small number $k$ of straight line segments.

Now, consider which subassemblies of $A$ could follow a given rigid motion $t$. Since a subassembly occupies space equal to the union of its parts, the motion $t$ causes a collision between a subassembly $S$ and $A \backslash S$ if and only if $t$ causes a collision between some part in $S$ and some part in $A \backslash S$.

Let $S$ be a subassembly removable along $t$. If a part $Q$ moved along $t$ collides with another part $P$ (left stationary), then we say that $P$ blocks $Q$ along $t$. If $P$ blocks $Q$, then either $P$ must be in the moved subassembly $S$ or $Q$ must not be in $S$. These constraints on membership in $S$ can be easily represented with a blocking graph [19]. The blocking graph of $A$ for motion $t$, written $G_{A}(t)$, is a directed graph with a node for each part of $A$ and an arc from node $Q$ to node $P$ exactly when $Q$ is blocked by $P$ along $t$. A subassembly $S$ can be removed with rigid motion $t$ if and only if no arcs in $G_{A}(t)$ connect nodes in $S$ to nodes in $A \backslash S$. Such a subassembly exists exactly when $G_{A}(t)$ is not strongly connected. ${ }^{2}$

The set of positions in which a part $Q$ collides with part $P$ is given by $P \ominus Q$, i.e., the Minkowski sum of $P$ and $-Q$ (see Subsection 3.2). Part $Q$ will collide with $P$ under a rigid motion $t$ exactly when the reference point of $Q$, moved along $t$, intersects $P \ominus Q$. To find the collisions between all pairs of parts for a single motion, all pairwise differences can be computed, taking the origin of the coordinate system to be the common reference point for all the parts. When the pairwise differences are superimposed, they partition the plane into regions, within which the set of pairs of parts colliding is fixed. A rigid motion $t$ causes the reference point to move through a sequence of regions, collecting constraints for the blocking graph of $t$. For further discussion see [13].

Figure 3 shows an assembly and the blocking graphs for two motions, one a translation up to the right, and the other a translation to the left then upward. For instance, part $B$ collides with part $C$ when translated up right, so the constraint $B \rightarrow C$ is present in the corresponding blocking graph. The blocking graph of the up-right translation is strongly connected, and in fact no subassembly can be removed along that path. For the two-step motion, the blocking graph is not strongly connected. Instead, there are no outgoing arcs from $\{B, C\}$ to $\{A\}$, so $B$ and $C$ may be removed rigidly in a motion to the left then upward.

We summarize our notation in Table 1.

\section{Partitioning with Two Translations}

Given an assembly $A$ of simple polygons, we wish to determine whether any subassembly $S$ of $A$ can be completely separated from the subassembly $A \backslash S$ by a finite translation of $S$ followed by an infinite translation of $S$. We describe a rigid motion consisting of two translations by

\footnotetext{
${ }^{1}$ Here "Y" denotes the set subtraction operator.

${ }^{2}$ A strongly connected component (or strong component) of a directed graph is a maximal subset of nodes such that for any pair of nodes $\left(n_{1}, n_{2}\right)$ in this subset, a path connects $n_{1}$ to $n_{2}$. A graph is strongly connected if it consists of one strong component.
} 

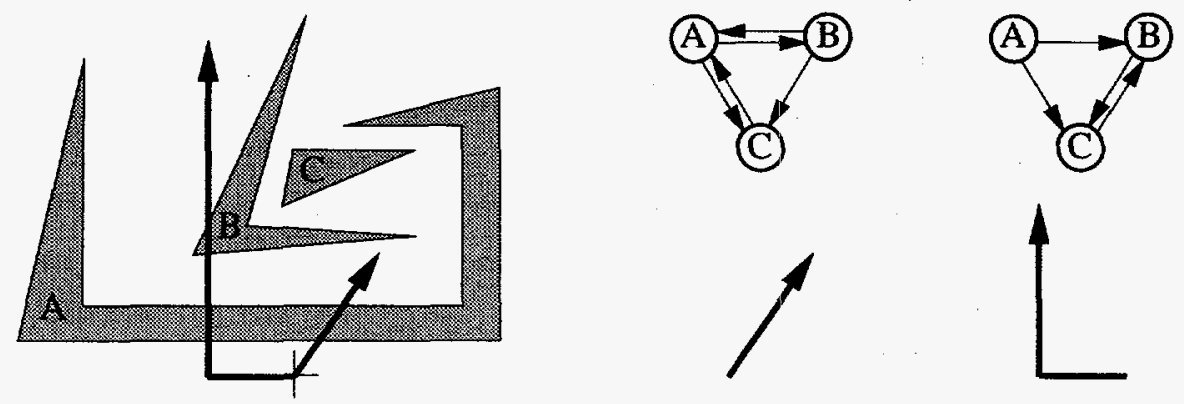

Figure 3: An assembly and its blocking graphs for two translational motions

\begin{tabular}{|c|l|l|}
\hline \hline symbol & \multicolumn{1}{|c|}{ description } & \multicolumn{1}{|c|}{ defined in } \\
\hline$n$ & number of parts in the assembly $A$ & \\
$N$ & overall number of features of parts in the assembly $A$ & \\
$K_{d}(m)$ & complexity of a $d$-dimensional arrangement of $m$ surfaces & Subsection 3.1 \\
$T_{d}(m)$ & time to compute a $d$-dimensional arrangement of $m$ surfaces & Subsection 3.1 \\
$P \ominus Q$ & the Minkowski sum of $P$ and $-Q$ & Subsection 3.2 \\
$S_{C}(M)$ & the central shadow of a polygon $M$ & Subsection 3.2 \\
$S_{\phi}(M)$ & the $\phi$-shadow of a polygon $M$ & Subsection 3.2 \\
$G_{A}(t)$ & the blocking graph for assembly $A$ along path $t$ & Subsection 3.3 \\
\hline \hline
\end{tabular}

Table 1: Summary of notation

a triple $t=(x, y, \phi)$, where $(x, y)$ is the displacement caused by the first translation, and $\phi$ is the direction of the second (infinite) translation. Since any motion $(x, y, \phi)$ will move the subassembly to infinity in direction $\phi$, we need only ensure that the motion is collision-free with the complement of the moved subassembly.

To solve the problem, we partition the $(x, y, \phi)$-space into cells such that the set of subassemblies that can follow a motion is fixed for all motions in a cell. First we derive the constraints arising from the first translation $(x, y)$, and then the constraints arising from a second translation given by $\phi$. Then we combine these sets of constraints to obtain the final three-dimensional arrangement, i.e., the subdivision of the $(x, y, \phi)$-space.

\subsection{The First Translation}

Consider now the constraints on subassemblies that can follow the first translation $(x, y)$ without collision, as $x$ and $y$ vary. The origin of the plane represents the null translation $(0,0)$, and we wish to calculate the critical curves in the plane at which the blocking graph for the first translation changes.

For any two parts $P$ and $Q$, the set of placements of $Q$ for which $Q$ intersects $P$ is the polygonal C-obstacle $C=P \ominus Q$. However, not all edges of $C$ are critical curves; once a translation enters $C$, the constraint $Q \rightarrow P$ will be present in the blocking graph for all translations further in the same direction. This leads us to compute the central shadow of 
$C, S_{C}(C)$. The edges of the central shadow $S_{C}(P \ominus Q)$ are (potential) critical curves for the first translation: the arc $Q \rightarrow P$ is present in blocking graphs for just those motions $(x, y) \in \operatorname{int}\left(S_{C}(P \ominus Q)\right)$ ending inside the shadow. ${ }^{3}$

We now superimpose the central shadows $S_{C}\left(P_{i} \ominus P_{j}\right)$ for all pairs of parts $\left(P_{i}, P_{j}\right)$. The boundary edges of the shadows determine a subdivision of the plane into regions (an arrangement of segments), such that for all points $(x, y)$ inside each region, $G_{A}((x, y))$ is fixed.

Figure 4 shows the C-obstacles (dotted lines) and the boundaries of the corresponding central shadows (solid) for each pair of parts of the assembly in Figure $3 . B / C$ is the obstacle for moving part $B$ and stationary part $C$; the obstacle $C / B$ is identical to $B / C$, rotated 180 degrees around the origin. The full arrangement for the first translation is shown at the bottom of Figure 4.

How many segments are there in this arrangement? Let the assembly $A$ have $n$ parts, and assume first that each part has at most some fixed number of vertices. (At this point we wish to emphasize the effect of the number of parts on the complexity. Below, in Subsection 4.4 we will give a refined analysis.) Thus the total complexity of the assembly is $O(n)$. By our assumption, the $\mathrm{C}$-obstacle for two parts can have an outer boundary with at most some fixed (constant) number of vertices, and the same holds for the boundary of the central shadow of such a polygon. For the consequent analysis, we need to know the maximum overall number of segments in the arrangement, which is $O\left(n^{2}\right)$ (Lemma 3.1). We denote this set of $O\left(n^{2}\right)$ segments by $S_{1}$.

\subsection{The Second Translation}

We now concentrate on the second, infinite translation. Such a translation is in fact a translation along a ray and it can be specified by three parameters $(x, y, \phi)$, where $(x, y)$ is the starting point of the ray and $\phi$ is its direction. We wish to partition the $(x, y, \phi)$-space into cells such that the set of subassemblies that can follow a motion along a ray is fixed for all the rays represented by the points in a cell. Note that, for the moment, we ignore the effect of the first translation on this subdivision.

We will define a collection of critical surfaces that induce the desired subdivision. We start by fixing a direction $\phi_{0}$ and considering a two-dimensional cross-section of the threedimensional space $(x, y, \phi)$, at $\phi_{0}$. At the fixed $\phi_{0}$, the critical curves are defined similar to the subdivision of the first translation, only this time we consider the $\phi_{0}$-shadows of the $\mathrm{C}$-obstacles rather than the central shadows. It is evident that inside a $\phi_{0}$-shadow of a Cobstacle, a new constraint is added, that does not exist out of that shadow. The collection of critical curves that determine the subdivision of the $\phi_{0}$-cross-section are the boundaries of the $\phi_{0}$-shadows of the C-obstacles.

To get the three-dimensional critical surfaces, we let $\phi$ vary, and let the boundaries of the $\phi$-shadows vary accordingly. The collection of critical surfaces is defined to be the union of the $\phi$-shadow boundaries for all $\phi \in[0,2 \pi)$. However, to simplify the analysis, we will consider a superset of the above collection. Namely, for every C-obstacle $D$, we will consider the surfaces traced by the set $S(D)$ of all the segments underlying the C-obstacle $D$ (see

\footnotetext{
${ }^{3}$ We say potential critical curves because we only care about edges where the transitive closure of the blocking graph changes, which is possibly a subset of the above edges. It might be the case, that considering only actual critical curves, that is, only edges where the transitive closure of the blocking graph changes, could lead to better bounds.
} 

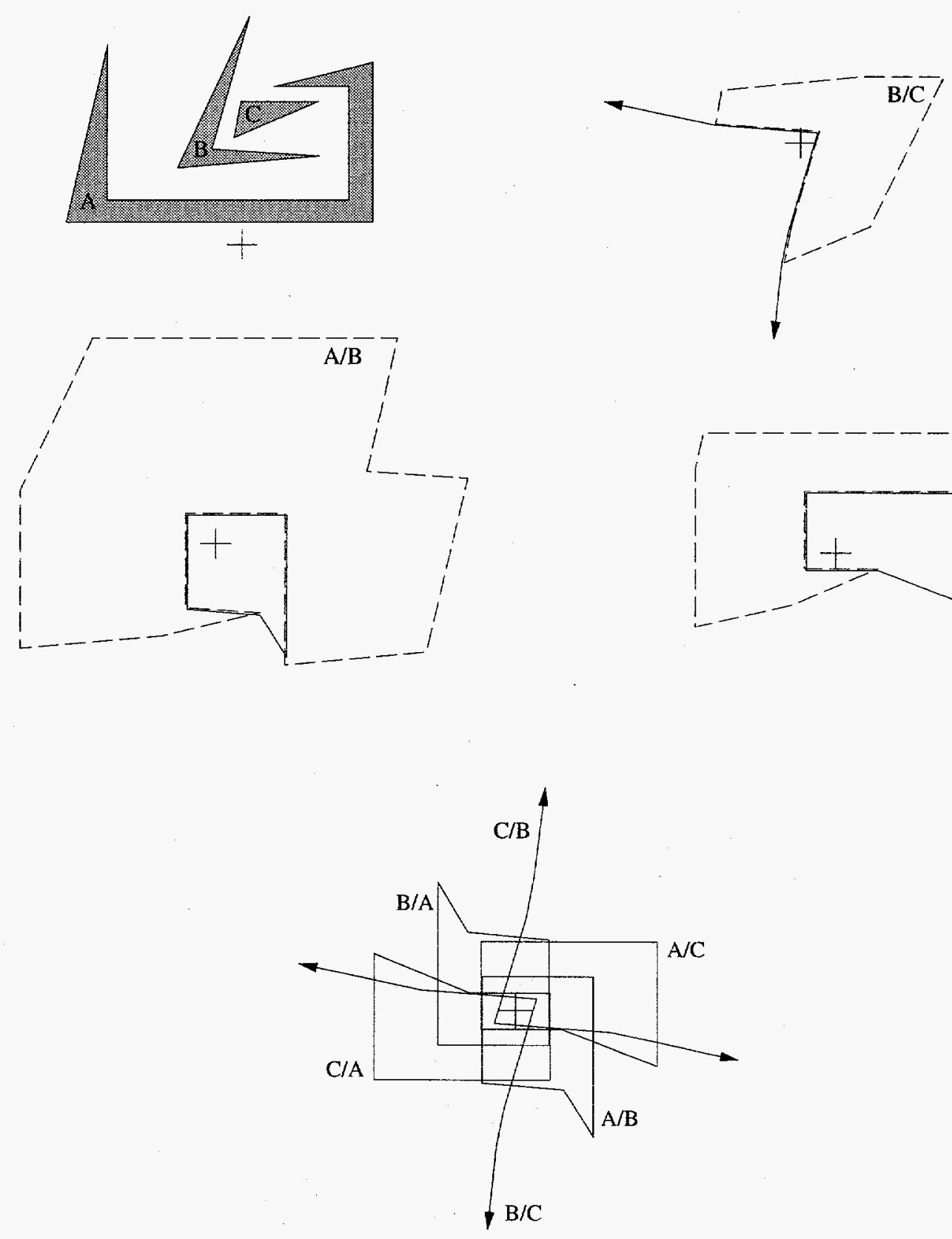

Figure 4: An assembly, central shadows for pairs of parts, and the arrangement for the first translation 
Subsection 3.2) as $\phi$ varies, together with surfaces traced by rays in the $\phi+180^{\circ}$ direction extended from endpoints of segments in $S(D)$ (that is, at a fixed $\phi$ we extend a ray from an endpoint of a segment in $S(D)$ in the $\phi+180^{\circ}$ direction, and let this ray change as $\phi$ changes; we consider the surface swept by this ray to be an additional critical surface and repeat this for every endpoint of any segment in $S(D)$ ). The resulting collection of surfaces is clearly a superset of the critical surfaces traced by the $\phi$-shadow boundaries. We denote this superset of critical surfaces by $\mathcal{S}_{2}$.

By the assumptions in Subsection 4.1, the number of surfaces induced by each C-obstacle is bounded by a constant. Since there are $O\left(n^{2}\right)$ C-obstacles (one for every pair of original parts), we conclude that $S_{2}$ consists of $O\left(n^{2}\right)$ critical surfaces, that partition the space $(x, y, \phi)$ into non-critical cells such that for all infinite translations $(x, y, \phi)$ inside each cell, the blocking graph $G_{A}((x, y, \phi))$ is fixed.

Figure 5 shows the $\phi$-shadows for the assembly of Figure 3 , and the resulting arrangement, for the upward translation $\phi=90^{\circ}$.

\subsection{Combining the Two Translations}

As stated in the beginning of this section, the triple $(x, y, \phi)$ can represent not only the second infinite translation, but in fact the two translations. A point $\left(x_{0}, y_{0}, \phi_{0}\right)$ represents a path of a subassembly that starts at the origin, moves to the point $\left(x_{0}, y_{0}\right)$ along a straight line segment, and then moves to infinity along a ray in the $\phi_{0}$ direction. We already have the set $\mathcal{S}_{2}$ of constraint surfaces that subdivides the space $(x, y, \phi)$ into non-critical cells, and we now refine this subdivision according to the constraints induced by the first translation. Consider a cross-section of the $(x, y, \phi)$-space at $\phi_{0}$. If we wish to add the constraints induced by the first translation, we should simply add the segments in the set $S_{1}$ to the arrangement. But this statement is valid for any $\phi$ value. Therefore, we extend each segment in $S_{1}$ into a vertical "wall" (in the $\phi$ direction) in the $(x, y, \phi)$-space. We will denote this collection of vertical walls, extended from $S_{1}$, by $\mathcal{S}_{1}$. Thus we have completed the subdivision of the space $(x, y, \phi)$ into non-critical cells, such that for any two-translation path $t=(x, y, \phi)$ in each cell the set of blocking constraints $G_{A}(t)$ is fixed.

The number of elements in $\mathcal{S}_{1}$ as well as in $\mathcal{S}_{2}$ is $O\left(n^{2}\right)$. These surfaces (or more precisely, surface patches) are clearly algebraic of bounded degree. By the discussion in Subsection 3.1 the maximum number of cells in a 3D arrangement induced by $m$ such surfaces, $K_{3}(m)$, is $O\left(m^{3}\right)$. Hence the maximum number of cells in the above subdivision is $O\left(n^{6}\right)$.

Finally, to solve the original problem, i.e., to find a subassembly that can be partitioned with two translations (if one exists), we proceed as follows. We compute the subdivision of the $(x, y, \phi)$-space by the surfaces in $\mathcal{S}_{1} \cup \mathcal{S}_{2}$, using an algorithm with running time $T_{3}\left(n^{2}\right)$ (see Subsection 3.1). For each cell produced by the algorithm, we compute the blocking graph $G_{A}(t)$ corresponding to a representative path $t$ for that cell. The blocking graphs for all cells in the arrangement can be computed incrementally in the following way. We begin by choosing a point $t=\left(x_{0}, y_{0}, \phi_{0}\right)$ in some cell $c_{0}$ of the arrangement; the blocking graph $G_{A}(t)$ can be easily computed by checking for inclusion of $\left(x_{0}, y_{0}\right)$ in the central shadows and $\phi_{0}$-shadows of the C-obstacles, in time proportional to the number of shadows, that is $O\left(n^{2}\right)$. (This step will be dominated by other steps of the algorithm, also in the refined analysis that we give below.) We then perform a systematic traversal of the arrangement, at each step moving from a cell to one of its neighbors. When we step through a critical surface, we either add or remove the constraint corresponding to that surface, depending on whether we are 

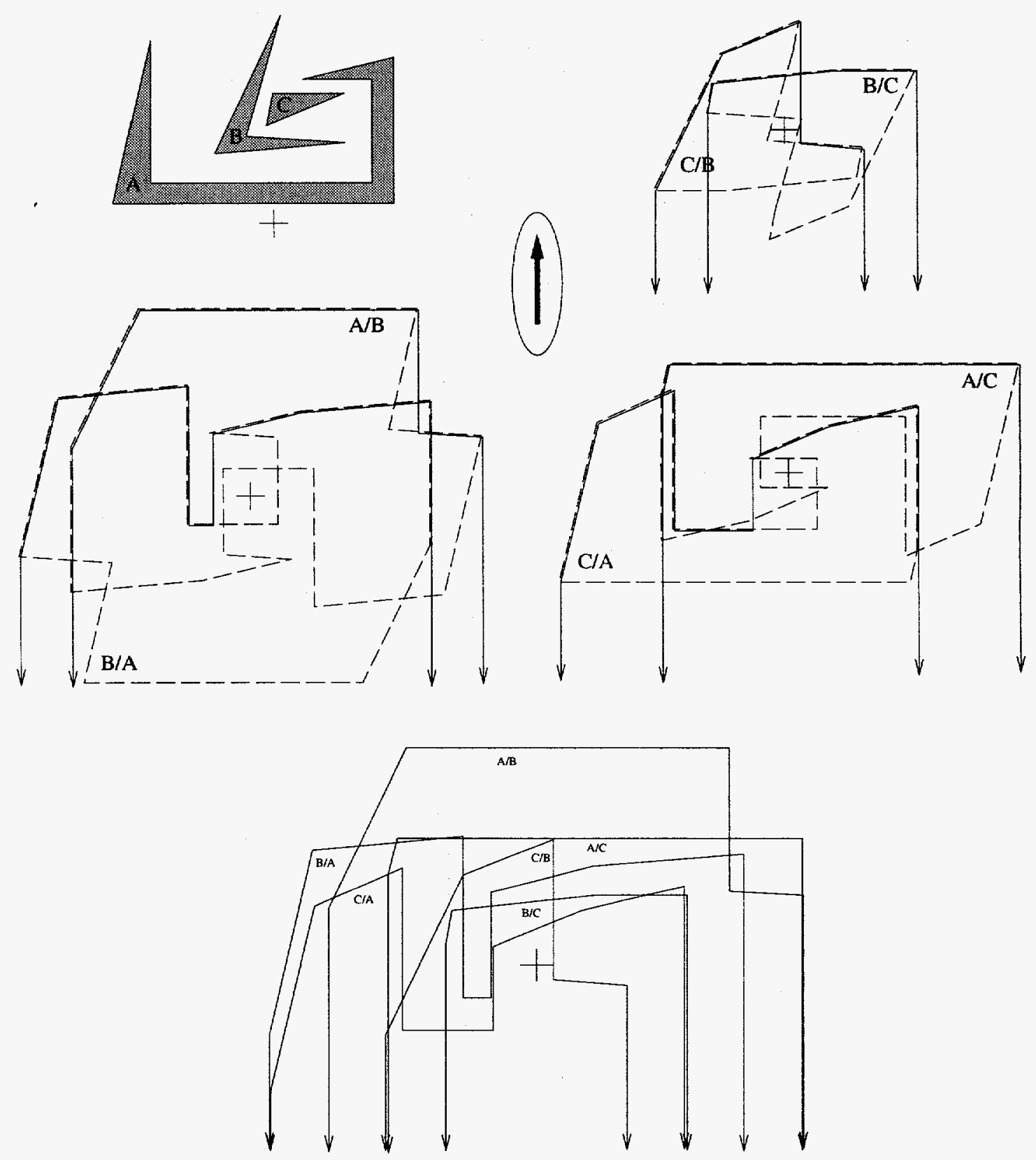

Figure 5: An assembly, $\phi$-shadows for pairs of parts when $\phi=90^{\circ}$, and a $2 \mathrm{D}$ slice of the arrangement for the second translation at $\phi=90^{\circ}$ 
entering a shadow or leaving it.

Since at most a constant number of constraints is added or removed at each step, ${ }^{4}$ all the blocking graphs can be computed in time proportional to the size of the arrangement, i.e., in time $O\left(n^{6}\right)$. Strong connectedness can be checked in time linear in the size of the graph, which in this case is bounded by $n^{2}$. Thus in an assembly of $n$ polygons, each having at most some constant number of vertices, the total time to find a subassembly removable by two translations is

$$
T_{3}(m)+K_{3}(m) O\left(n^{2}\right), \text { where } m=O\left(n^{2}\right) .
$$

We summarize the discussion above in the following theorem.

Theorem 4.1 Given a planar assembly consisting of $n$ disjoint simple polygons, each having at most some constant number of vertices, it can be determined in $O\left(n^{8}\right)$ time whether there is a subassembly that can be removed along a path consisting of a single finite translation followed by a translation to infinity. The algorithm outputs both the labels of the parts in the removable subassembly and the specifications of the path.

\subsection{Refined Analysis}

In this subsection we introduce another parameter into the analysis of the running time of our algorithm, and also indicate several points where the algorithm may be improved.

Let the assembly $A$ we wish to partition consist of $n$ parts (as before) and let $N$ be the total number of vertices of all the parts together. Denote the number of vertices of part $P_{i} \in A$, by $n_{i}$. As discussed in Subsection 3.2 (see also Lemma 3.1), the crucial parameter ${ }^{5}$ for each C-obstacle defined by two parts $P_{i}, P_{j}$, is the number of segments underlying the Minkowski sum $P_{i} \ominus P_{j}$, which is $O\left(n_{i} n_{j}\right)$. Hence in both sets $\mathcal{S}_{1}$ and $\mathcal{S}_{2}$ of the analysis in this section, the overall number of surfaces is

$$
\sum_{i \neq j} O\left(n_{i} n_{j}\right)=O\left(N^{2}\right)
$$

Therefore we have

Theorem 4.2 Given a planar assembly consisting of $n$ disjoint simple polygons, having a total of $N$ vertices altogether, it can be determined in $O\left(n^{2} N^{6}\right)$ time whether there is a subassembly that can be removed along a path consisting of a single finite translation followed by a translation to infinity. The algorithm outputs both the labels of the parts in the removable subassembly and the specifications of the path.

The bound above assumes a worst case combinatorial bound $O\left(\mathrm{~m}^{3}\right)$ for an arrangement of $m$ surfaces in 3 -space. It might be that the underlying arrangement does not achieve this bound. To exploit the potential "sparseness" of the arrangement one can employ an outputsensitive algorithm for computing the arrangement, like the one devised by de Berg et al. ${ }^{6}$ [4].

\footnotetext{
${ }^{4}$ We are assuming here general position of the parts in the arrangement. Certain technical modifications to the algorithm will be necessary to handle "degenerate" assemblies.

${ }^{5}$ For practical use, one may gain a lot from computing only the boundary rather than using all the segments. This may affect the constant factor in our analysis.

${ }^{6}$ One does not need all the machinery of [4] in this case. All that is needed here is the so-called "first step decomposition" of the arrangement. Although [4] deals with triangles in space, the algorithm for computing the required decomposition can be easily extended to the arrangements studied in this paper.
} 
It may appear that checking each blocking graph independently for strong connectedness is rather wasteful, since each graph differs from the previous one by at most a single constraint. In related work, Khanna, Motwani, and Wilson have shown the following: given a directed graph with $n$ nodes, and a "long" (compared to $n$ ) sequence of edge insertions and deletions to that graph, the strong connectedness of all resulting graphs can be determined in amortized time $O\left(n^{1.46}\right)$ per graph [11]. The method groups the sequence of graphs into phases and pre-processes the common sub-graph for each phase. The method applies directly to our problem, resulting in the following theorem.

Theorem 4.3 Given a planar assembly consisting of $n$ disjoint simple polygons, having a total of $N$ vertices altogether, it can be determined in $O\left(n^{1.46} N^{6}\right)$ time whether there is a subassembly that can be removed along a path consisting of a single finite translation followed by a translation to infinity. The algorithm outputs both the labels of the parts in the removable subassembly and the specifications of the path.

\section{Partitioning with a Bounded Number of Translations}

We now consider partitioning an assembly along a path consisting of a small number $k$ of translations $m_{1}, m_{2}, \ldots, m_{k}$. There are $2 k-1$ degrees of freedom in specifying the path $t$. Two parameters $\left(x_{i}, y_{i}\right)$ specify the endpoint of each of the first $k-1$ moves, and one parameter $\phi$ specifies the direction of the last (infinite) move. Hence we will examine the $k$-translation problem in a $2 k-1$-dimensional space with coordinates $\left(x^{(1)}, y^{(1)}, \ldots, x^{(k-1)}, y^{(k-1)}, \phi\right)$.

We distinguish three different types of moves along the path $t$ : the first move $m_{1}$ starting at $(0,0)$, the last (infinite) move $m_{k}$, and the intermediate moves $m_{2}, \ldots, m_{k-1}$. As we will show below, the analysis of the first and the last moves is similar to the analysis in Subsections 4.1 and 4.2 respectively. Thus we start by analyzing the contribution of constraint (hyper)surfaces by an intermediate move $m_{i}$.

Consider the four-dimensional space with coordinates $\left(x^{(i-1)}, y^{(i-1)}, x^{(i)}, y^{(i)}\right)$, where every point represents an intermediate translational move in the plane from $\left(x^{(i-1)}, y^{(i-1)}\right)$ to $\left(x^{(i)}, y^{(i)}\right)$. We wish to subdivide the space $\left(x^{(i-1)}, y^{(i-1)}, x^{(i)}, y^{(i)}\right)$ into non-critical cells, such that the blocking graph corresponding to the moves represented by all the points inside the cell is the same. It can be easily shown that every feature of a C-obstacle contributes at most a fixed (constant) number of critical three dimensional hypersurfaces, all algebraic of bounded degree.

The analysis of constraint curves induced by the first motion in the $2 \mathrm{D}$ space with coordinates $\left(x^{(1)}, y^{(1)}\right)$ is the same as the analysis in Subsection 4.1. Similarly, the analysis of constraint surfaces induced by the last move in the 3D space with coordinates $\left(x^{(k-1)}, y^{(k-1)}, \phi\right)$ is the same as the analysis in Subsection 4.2. Our goal now is to put all the constraints together in the $2 k-1$-dimensional space with coordinates $\left(x^{(1)}, y^{(1)}, \ldots, x^{(k-1)}, y^{(k-1)}, \phi\right)$. We use the guidelines that we have used in Subsection 4.3. For example, consider a 3D constraint hypersurface defined above in the space $\left(x^{(i-1)}, y^{(i-1)}, x^{(i)}, y^{(i)}\right)$. This $4 \mathrm{D}$ space can be viewed as a $4 \mathrm{D}$ cross-section of the underlying $2 k-1$ dimensional space, where all but the four coordinates $\left(x^{(i-1)}, y^{(i-1)}, x^{(i)}, y^{(i)}\right)$ are fixed. Moreover, the same 3D constraint prevails for every other $4 \mathrm{D}$ cross-section as above of the $2 k-1$-dimensional space. Hence, we extend every critical hypersurface defined in the $k$ subspaces $\left(x^{(1)}, y^{(1)}\right),\left(x^{(1)}, y^{(1)}, x^{(2)}, y^{(2)}\right), \ldots,\left(x^{(k-1)}, y^{(k-1)}, \phi\right)$ into a $2 k$ - 2-dimensional surface in the space $\left(x^{(1)}, y^{(1)}, \ldots, x^{(k-1)}, y^{(k-1)}, \phi\right)$, in the obvious manner. 
Let $\mu$ denote the overall number of features (vertices and edges) in all the $O\left(n^{2}\right)$ original C-obstacles defined earlier. Recall that, by the analysis in Subsection 4.4, $\mu=O\left(N^{2}\right)$. Note that each of the $k$ moves contributes $O(\mu)$ critical $2 k-2$ dimensional surfaces. Therefore, the number of non-critical cells in the subdivision of the space $\left(x^{(1)}, y^{(1)}, \ldots, x^{(k-1)}, y^{(k-1)}, \phi\right)$, is $O\left((k \mu)^{2 k-1}\right)$.

To compute a subassembly that is separable by $k$ translations we employ an algorithm to produce all the cells in the above $2 k$-1-dimensional arrangement, and for each cell we check the corresponding blocking graph for strong connectedness.

Theorem 5.1 Given a planar assembly consisting of $n$ disjoint simple polygons, having a total of $N$ vertices altogether, it can be determined in $O\left(\left(k N^{2}\right)^{2 k-1} n^{2}\right)$ time whether there is a subassembly that can be removed along a path consisting of at most $k$ translations where the last translation extends to infinity. The algorithm outputs both the labels of the parts in the removable subassembly and the specifications of the path.

Proof. The actual computation of the arrangement can be carried out in time roughly $O\left(\left(k N^{2}\right)^{2 k-1}\right)$ (see Subsection 3.1) and hence it is negligible in comparison with the strong connectivity check for all the cells. The $2 k-1$ dimensional arrangement is determined by $O\left(k N^{2}\right)$ hyper surfaces, and therefore the maximum number of cells in it is $O\left(\left(k N^{2}\right)^{2 k-1}\right)$. In each cell we spend time $O\left(n^{2}\right)$ to check for strong connectivity, and the bound $O\left(\left(k N^{2}\right)^{2 k-1} n^{2}\right)$ follows.

\section{Conclusions}

In this paper, we have extended the non-directional blocking graph (NDBG) framework for the assembly partitioning problem devised by Wilson [18] to the case of a compound removal path consisting of a small number of translations. Our new result builds on two existing concepts: the concept of the NDBG [18] and the "interference diagram" [13]. Previously, NDBGs were studied only for simple types of motions, while it was not clear how to use the interference diagram efficiently in order to solve the partitioning problem.

The major open problem that our paper raises is to improve the running time of the algorithms presented in it. Some possible directions for improvement are suggested in Subsection 4.4. Another related question, which applies to other instances of the NDBG framework as well, is the following: We compute a collection of $n(n-1)$ C-obstacles. However, this collection of C-obstacles is induced by only $n$ parts. Can we exploit this fact to improve the running time of our algorithm, or of other algorithms that deal with the NDBG? We are currently investigating this question. Another direction that we are currently exploring is the introduction of randomization into the algorithm, in order to try to avoid the computation of the entire arrangement in situations where this is not necessary.

In this paper we have confined ourselves to translational motion along straight line segments. It is desirable to extend the framework to compound motions that consist of more elaborate basic moves, particularly including rotations. 


\section{References}

[1] E. M. Arkin, R. Connelly, and J. S. B. Mitchell. On monotone paths among obstacles, with applications to planning assemblies. In Proc. of the 5th ACM Symp. on Computational Geometry, pages 334-343, 1989.

[2] S. Basu, R. Pollack, and M.-F. Roy. A new algorithm to find a point in every cell defined by a family of polynomials. In B. Caviness and J. Johnson, editors, Quantifier Elimination and Cylindrical Algebraic Decomposition. Springer-Verlag. To appear.

[3] J, Canny. Personal communication.

[4] M. de Berg, L. Guibas, and D. Halperin. Vertical decompositions for triangles in 3-space. In Proc. 10th Annu. ACM Symp. Comput. Geom., 1994.

[5] H. Edelsbrunner. Algorithms in Combinatorial Geometry. Springer-Verlag, 1987.

[6] L. Guibas and M. Sharir. Combinatorics and algorithms of arrangements. In J. Pach, editor, New Trends in Discrete and Computational Geometry, pages 9-36. Springer, 1993.

[7] D. Halperin. Algorithmic motion planning via arrangements of curves and of surfaces. $\mathrm{PhD}$ thesis, Dept. of Computer Science, Tel-Aviv Univ., July 1992.

[8] L. S. Homem de Mello and S. Lee, editors. Computer-Aided Mechanical Assembly Planning. Kluwer Academic Publishers, 1991.

[9] A. Kaul, M. A. O'Connor, and V. Srinivasan. Computing Minkowski sums of regular polygons. In Proc. 3rd Canad. Conf. Comput. Geom., pages 74-77, 1991.

[10] L. Kavraki, J.-C. Latombe, and R. H. Wilson. On the complexity of assembly partitioning. Information Processing Letters, 48(5):229-235, 1993.

[11] S. Khanna, R. Motwani, and R. H. Wilson. Dynamic strong connectivity with a lookahead. In preparation, 1994.

[12] J.-C. Latombe. Robot Motion Planning. Kluwer Academic Publishers, 1991.

[13] T. Lozano-Pérez and R. H. Wilson. Assembly sequencing for arbitrary motions. In Proc. of the IEEE Intl. Conf. on Robotics and Automation, volume 2, pages 527-532, 1993.

[14] J. S. B. Mitchell. Personal communication, December 1990.

[15] R. Pollack and M. Roy. On the number of cells defined by a set of polynomials. C.R. Acad. Sci. Paris, (t. 316, Série I):573-577, 1993.

[16] R. Pollack, M. Sharir, and S. Sifrony. Separating two simple polygons by a sequence of translations. Discrete and Computational Geometry, 3:123-136, 1988.

[17] G. T. Toussaint. Movable separability of sets. In G. T. Toussaint, editor, Computational Geometry. Elsevier, 1985.

[18] R. H. Wilson. On Geometric Assembly Planning. PhD thesis, Stanford Univ., March 1992. Stanford Technical Report STAN-CS-92-1416. 
[19] R. H. Wilson and J.-C. Latombe. Geometric reasoning about mechanical assembly. Artificial Intelligence, 71(2), 1994. 
Distribution:

MS $9018 \quad$ Central Technical Files, 8523-2

MS 0899 Technical Library, 13414

MS 0619 Technical Publications, 13416

MS 0100 Document Processing for DOE/OSTI, 7613-2

MS $0951 \quad$ Randy Wilson, 2121 